\title{
Agôn
}

Revue des arts de la scène

$\varnothing \mid 2007$

En quête du sujet

\section{La quête identitaire du sujet dans le théâtre contemporain africain}

Alice Carré

\section{OpenEdition}

1 Journals

Édition électronique

URL : http://journals.openedition.org/agon/329

DOI : 10.4000/agon.329

ISSN : 1961-8581

\section{Éditeur}

Association Agôn

\section{Référence électronique}

Alice Carré, «La quête identitaire du sujet dans le théâtre contemporain africain », Agôn [En ligne], $\varnothing \mid$ 2007, mis en ligne le 11 décembre 2007, consulté le 17 avril 2020. URL : http:// journals.openedition.org/agon/329; DOI : https://doi.org/10.4000/agon.329

Ce document a été généré automatiquement le 17 avril 2020

Association Agôn et les auteurs des articles 


\title{
La quête identitaire du sujet dans le théâtre contemporain africain
}

\author{
Alice Carré
}

Je traîne à la queue d'une tribu perdue, comme un animal des savanes hanté par le rythme d'un autre troupeau.

1 Sony Labou Tansi, romancier et dramaturge congolais, marque l'ouverture de la littérature africaine sur le monde. Assumant la vacuité d'une identité à jamais perdue, définitivement réinventée par la colonisation, celui qu'on appelle le «BlackShakespeare " rompt le premier avec la représentation d'une Afrique fermée, îlot préservé des évolutions du monde.

2 Dans les années 1990, sur ses traces, de jeunes auteurs se tournent vers le théâtre avec la conviction que leurs pièces entreront sur la scène des écritures contemporaines. Le théâtre africain se confronte résolument à autrui, mettant fin à une prétendue spécificité colportée par l'imaginaire colonial fait de marabouts, de griots et de tamtams. L'écriture veut déranger les préjugés occidentaux, et situer l'identité africaine là où on ne l'attend pas. Cependant, cette vision occidentale est elle-même fantasmée par les auteurs d'une nouvelle génération africaine qui se laissent emprisonner dans une image présumée d'eux-mêmes, comme si la perception européenne de l'Afrique n'avait pas changé depuis le $\mathrm{XIX}^{\mathrm{e}}$ siècle. La problématique de l'identité qui traverse leurs œuvres se fonde sur un complexe d'infériorité : les écrivains effacent mal la cicatrice saillante de la période coloniale, bien qu'ils se revendiquent de la génération des Indépendances, libérée des questionnements identitaires imposés par la domination européenne. Certes, ces dramaturgies surprennent, refusent de mettre la colonisation au premier plan, s'opposent à toute approche normative de l'identité africaine, mais derrière chaque personnage se dresse le spectre de la colonisation.

3 L'étude de la question identitaire dans ce théâtre s'appuie sur quatre auteurs : Koffi Kwahulé, metteur en scène et dramaturge d'origine ivoirienne né en 1956, Caya Makhélé, dramaturge, journaliste et romancier congolais né en 1954, Koulsy Lamko, né en 1959 au Tchad, enseignant au Rwanda, Gustave Akakpo, auteur, conteur, illustrateur 
et acteur né en 1974 au Togo. Ces auteurs ont choisi d'écrire en français, langue de la métropole : "pour ne pas subir cette langue, il faut que je la fasse sonner autrement ", explique Koffi Kwahulé. La lutte engagée avec cette langue d'abord imposée puis réappropriée, rejoint celle plus globale menée contre la perte de soi. mealtérité

7 - Le sujet finit par se saisir uniquement dans l'image que l'autre lui renvoie, ou telle qu'il croit la deviner. L'identité s'objective et crée une image complexée d'elle-même. Mettre le spectateur en scène apparaît comme une solution pour agir sur ses propres névroses, et de détruire en l'autre toute possibilité d'adopter une vision normative.

La quête de soi et la tentative de reconstruire une identité guide le théâtre des quatre auteurs. Mais cette quête sait d'avance que son but n'est que fictif, car l'identité ne permet jamais de se cerner entièrement soi-même, de se posséder; elle n'est qu'un processus mouvant, brisant toute certitude et toute délimitation.

\section{Vacance du sujet}

Dans ces dramaturgies, la caractéristique première du personnage est d'errer en scène plutôt que d'y agir. À la recherche de lui-même, il souffre d'un défaut d'identité et apparaît souvent sous la figure du vagabond ou du clochard, comme le personnage principal de La Fable du cloître aux cimetières de Caya Makhélé, Makiadi, « ex-colleur de timbres au ministère de la santé publique, aujourd'hui clochardisé ${ }^{1}$ ». Les premiers mots présentent sa décrépitude physique, jouant sur le dégoût pour le spectateur: «Makiadi crache abondamment dans ses mains et se fait un brin de toilette, en chantonnant $^{2} »$.

La scène quotidienne met en évidence un personnage répugnant et travaille la veine comique du trivial :

Ma faune intestinale s'arroge le droit de crier en se serrant la ceinture. Ils sont tous là, asticots, lombrics, vermines, vermisseaux, ascaris, filaires, ténias, vers-coquins, vers solitaires. Tous réfugiés politiques dans mon ventre. ${ }^{3}$

11 Le personnage est réduit à un corps désolidarisé, à des organes rongés et parasités par des corps étrangers, métaphore de l'impact de la colonisation sur l'intime. Makiadi est menacé par l'éclatement, l'incapacité d'exister comme un tout, de se saisir lui-même. Sa vision de lui-même est parcellaire et incomplète : « Aïe, coupure d'un sale miroir qui ne me laisse voir que ma tête et pas le reste de mon corps ${ }^{4} »$. Le corps, comme le miroir brisé, est multiple, l'image de soi est complexe et le sujet épars.

Cette image du corps dégradé n'est pas sans rappeler les êtres de Beckett, caractérisés par un corps souffrant, tel Hamm dans Fin de partie, paralysé, aveugle, réduit à l'utilisation d'une sonde urinaire, ainsi que ses parents Nell et Nagg habitants de deux poubelles, ou encore Clov, réduit à une activité permanente car il ne peut pas s'asseoir. On remarque un héritage et une même symbolique : au corps dégradé correspond une impossibilité d'action et d'existence. Le début du Destin d'Ariane de Makhélé rend 
explicite cette relation entre existence et dislocation. Dans la première scène intitulée "Qui-suis-je?", Ariane immobile laisse tomber ses paniers, ouvre une armoire, une pluie de poupées se déverse sur elle, elle fredonne: "Coco cassé, bébé brisé, poupée déchirée ${ }^{5}$ ». Le débris du corps de la poupée correspond par métonymie à l'identité morcelée du personnage. À corps brisé, image de soi fractionnée et sujet disloqué.

Vacant, le sujet l'est aussi par sa passivité, et son refus d'entrer en adéquation avec le monde. Pensons au vieillard de Tout bas... si bas qui a élu domicile dans un arbre et refuse d'en descendre, de toucher le sol d'un monde honni. Le héros de la Fable du cloitre des cimetières quant à lui, ne peut intervenir dans sa propre histoire qu'à contre courant. La pièce travaille l'opposion entre une activité hors scène très intense, bruyante et l'image fixe du personnage au centre de la scène, seul, immobile, passif. Le personnage se laisse porter par les jours, jusqu'au moment où une lettre envoyée par une mystérieuse femme morte le ramène à la vie. Celle-ci lui déclare son amour et lui apprend que lui seul peut la ramener dans le monde des vivants. Le héros entre donc en quête du monde des morts, imitant comme un double inversé le personnage d'Orphée à la recherche de son Eurydice. La réécriture du mythe indique ici une vision cynique du monde. Il n'y a plus de héros possible : Orphée ne connaît pas le chemin des Enfers, et ne sait pas s'il reconnaitra son Eurydice si elle se présente à lui. Il se trompe d'abord de lieu, et, s'étant glissé dans un cercueil, atterit à la morgue. Enfer désenchanté et aseptisé : Cerbère s'est changé en gardien en blouse blanche. Faux départ de l'intrigue, stratégies inadéquates choisies par le héros, la pièce est construite sur une succession d'actions déceptives. Et pour finir, on s'aperçoit que l'histoire d'amour n'était qu'une duperie adressé à Makiadi pour le forcer à se trouver lui-même. À la dernière page, lorsque l'on comprend que le gardien de la morgue a tout combiné pour léguer sa place à Makiadi, on est obligé de reconsidérer la pièce. La véritable quête de Makiadi n'était donc pas de ramener la femme aimée à la vie mais de s'y ramener lui-même : «il est temps que je redevienne humain ${ }^{6}$ ", déclare-t-il au début de la pièce.

La pièce apparaît alors comme un parcours initiatique, une quête identitaire. Les titres didascaliques ${ }^{7}$ rappellent les paroles d'un conteur narrant le destin du héros : "Ici commence la métamorphose de Makiadi ${ }^{8} »$ indique l'un d'eux. Et Ogba, le dieu diable rappelle : "Ne pensez-vous pas que nous vivons le siècle des identités? ", en tendant à Makiadi un masque pour changer d'apparence. Durant toute la pièce, Makiadi change de visage. Il se cherche d'abord dans le reflet d'un miroir brisé, se travestit en femme, revêt la soutane d'un pasteur. À chaque transformation, Makiadi a beau clamer haut et fort qu'il est le clochard du début, personne ne le reconnaît. "J'ai l'habitude de ne pas être reconnu $u^{9}$ ", se morfond-t-il. Car la reconnaissance par autrui est la condition sine qua non pour exister. Il n'y a donc de différence pour personne dans cette pièce entre être et apparence, et ce monde déréglé semble faire le procès d'une société pas si lointaine, où l'africain se sent enfermé. Toutefois, l'identité du personnanage se révèle de plus en plus solide à travers toutes ces péripéties. Et sur une lettre envoyée par la présumée femme morte apparaît la nécessité d'avoir le courage d'exister soi-même :

Je suis contente que tu aies retrouvé ton masque de clochard. Il te va tellement bien. Sais-tu qu'ici mes amies et moi l'appelons le masque de l'héroïsme. ${ }^{10}$

L'héroïsme est donc le courage d'être soi-même et de s'affirmer face à autrui sans se contrefaire. Après avoir accepté de se traverstir sous toutes les formes le personnage redevient lui-même mais en acceptant le monde dans lequel il vit, puisque à la fin, il occupe un place dans la société : gardien de la morgue. 
16 Ainsi, le destin métaphorique du personnage retrace l'abandon de l'image idéale d'une Afrique embaumée et l'acceptation d'un temps présent. Motéma, la femme morte, image de l'Afrique d'antant, morte écrasée sous un train, symbole de la fulgurance du monde moderne, enjoint l'africain de se remettre à vivre.

\title{
L'invention du sujet par l'autre
}

\author{
Je sais, je sais, tu ne t'appelles pas Stan. Mais, moi, \\ je veux t'appeler Stan [...] ça me booste de \\ t'appeler Stan [...] tu t'appelles Stan et tu la \\ boucles! OK ? ${ }^{11}$
}

Big Shoot de Koffi Kwahulé suscite un désarroi initial du lecteur : pas de distribution des personnages, et, dans le corps du texte, pas de didascalie, pas de sujet de parole. Un saut de ligne permet de présenter le changement d'énonciation, blanc typographique plus ou moins visible flottant dans un dialogue décousu, tirant vers le monologue. Le personnage n'est presque pas repérable dans le texte. Pour un spectateur, la situation est à peine plus claire.

"Dans une cage de verre carrée, deux hommes ${ }^{12}$ ", précise la didascalie initiale, mais le début paradoxal de la pièce ne permet pas d'associer les personnages à une situation ni de les mettre en action. La pièce débute par une série d'insultes proférées à un être immobile. Discours déroutant, mi en anglais, mi en français, où l'un hurle sur l'autre, frappe, sans raison apparente autre que la gratuité et la domination. L'un des personnages cherche à faire raconter à l'autre un souvenir qui visiblement n'a jamais existé, à l'inventer par ses questions. Mais si l'autre a l'air de mentir, il frappe. Le personnage est décrit d'emblée comme un être inventé de toutes pièces. Le texte mime ouvertement leur artificialité.

19 Ici encore, les personnages entrent sur scène indéfinis, et s'inventent l'un l'autre. On ne saura rien d'eux, pas même leur identité réelle. Dans ce processus, l'autre intervient comme une extériorité violente, qui détermine l'essence de celui qui est en face. La violence verbale et la domination président à ce processus. Le rapport entre le bourreau et la victime de Stan et de Monsieur permet une dépossession totale de l'autre. La victime est créée par son oppresseur : son nom, Stan, ce qu'il vient de faire, ses souvenirs, et cette action anodine sur laquelle on l'interroge. Le bourreau va jusqu'à fabriquer le crime de Stan, question après question, il reprend la narration : Stan aurait saisi un pique feu, l'aurait introduit dans le sexe de sa victime, puis aurait abusé d'elle toute une nuit après l'avoir tuée. Stan acquiesce d'abord, s'y oppose ensuite, puis reconnaît pour ne plus en entendre parler, avoir profané le corps de cette femme pendant des heures. Mais tout au long de ce face à face pervers, Monsieur a méthodiquement construit le crime de Stan. Il l'accuse alors d'avoir menti, et celui-ci le reconnaît. Nous sommes alors face à l'énigme de ce personnage venu se faire abattre sans raison. Sans raison, il l'avoue lui-même. Sauf peut-être la volonté de «sortir de l'anonymat ", d'exister par le regard autrui. Car peut-être que dans ce mensonge seul Stan aura existé aux yeux du public, masse collective se repaissant de son existence.

Le sujet chez Koffi Kwahulé naît de la violence. Comment ne pas rapprocher ce processus de celui de la colonisation, où l'occidental a méticuleusement oeuvré à inventer l'autre, à le renommer d'un nom occidental selon le bastion dans lequel il servait? Comment ne pas penser aux zoos humains, où les expériences 
anthropologiques faisaient du noir un objet pittoresque et de ses coutumes un ensemble de clichés à regarder en se pavanant au jardin des plantes? Si ce passé là rejaillit dans le sujet de théâtre, c'est de manière terriblement actuelle, sur ce qu'on suppose être un plateau de télévision, dans une réalité accrue de notre monde. Plusieurs indices portent à croire que Monsieur est un animateur de jeu télévisé et que Stan est un participant, une victime consentante qui accepte que l'on mette fin à ses jours en direct. Il est soumis à un interrogatoire public dont la fin annoncée sera une mise à mort. Rien n'est fait pour le clarifier, mais «la cage de verre, [...] nettoyée à l'ammoniaque, clean ${ }^{13}$ ", transparence de la bestialité humaine, trace du voyeurisme du reality show, ainsi que les références au public: "venus de partout, chaque fois plus nombreux », nous y conduisent. Le show est même présenté explicitement : «Et quand le public s'en mêle, là, Stan, les si, les ça, les parce que, les quand-même... non, non... Plus le temps pour basculer... The show must go on $!^{14} »$. Au fond, ce sujet qui est observé sur un plateau de télévision comme entre deux lames de verre apparaît comme une tortueuse réflexion sur la condition humaine. Le sujet Stan est observé de la naissance à la mort; sur un plan symbolique, il nait: univers aseptisé qui pourrait rappeler l'univers médical, cage en verre qui pourrait être une couveuse, Monsieur nomme Stan de manière purement arbitraire. Il apprend la parole - ou plutôt ce qu'il doit dire, il est dressé au langage. Stan procède par étoffement progressif de sa parole, par répétition de ce qui vient d'être dit.

Le mythe de Caïn et Abel régit toute la pièce en toile de fond. Dès le début de la pièce on voit le premier homme chanter un passage de la Genèse :

Then the Lord said to Caïn,

« Where is your brother Abel?»

"I don't know », he replied.

«Am I my brother's keeper ? $^{15}$ "

Le mythe relie aux origines de l'humanité, et au crime initial qui structure la société humaine dans l'imaginaire judéo-chrétien. Le crime chez l'homme apparaît au stade embryonnaire, expliquait Kristian Fredrik, qui présentait une mise en scène de la pièce à Montréal en avril 2007: "Déjà sous forme de cellule, nous combattons ceux qui pourraient nous écarter de la vie ». Chaque être humain est fratricide. Inventer l'autre c'est le tuer, c'est se placer en sujet agissant et faire de l'autre l'objet passif de ses fantasmes. Mais cette supériorité défaille, car Monsieur ne peut exister sans cet autre sur qui il projette son autorité, et que son être est d'une profonde vacuité. Lui aussi aspire à la définition, mais c'est lui qui en pose les termes :

Je suis calme, Stan, pas vrai?

Oui très calme.

Tu ne peux pas dire que je ne suis pas tolérant? Reconnais-le!

Vous êtes tolérant.

Je me montre humain... Je suis quand même humain, Stan?

Vous êtes humain. ${ }^{16}$

Cependant, ces questions / réponses de Monsieur, où Stan répète habilement ce que l'on veut entendre, révèlent une faille. Le bourreau s'accroche désespérément à ces réponses sans lesquelles il sombre dans l'inconscience de lui-même : la répétition du mot «humain» ainsi que les points de suspension l'indiquent. La pièce va vers un progressif retournement de situation, qui gravite autour de l'angoisse de «Monsieur»de ne pas pouvoir se définir. Monsieur n'a pas d'autre nom qu'une manière de s'adresser à lui, Monsieur n'existe pas sans interlocuteur, Monsieur a une 
« sorte d'accent » quand il parle anglais. Cette obsession le suit tout au long de la pièce : "Vous avez de l'accent Monsieur./ Une sorte d'accent terrible et compact ${ }^{17}$. " Même sur la chaise d'exécution, alors qu'il doit presser la gâchette et jouer à la roulette russe, Stan le soutient avec calme. L'homme reste seul avec le cadavre présumé de Stan et n'a pas avancé dans la quête de lui-même. Il rumine son accent terrible et compact, et reste face au vide. Si le sujet n'est pas reconnu par l'autre, si la définition que l'on a de soi n'est pas acceptée par un tiers, elle n'est pas valable, et l'on tombe dans les limbes de la perte de soi.

Cette définition tortionnaire de l'identité apparaît sur un plateau télévision. Le personnage est dépossédé de lui-même au profit d'une société avide de vie privée et d'un système en quête d'audimat. Le participant est un objet sur lequel chacun peut exercer ses vices, projeter ses envies de domination. À tous les niveaux, on existe à travers l'autre, et c'est contre cette existence forcée que s'insurgent les dramaturges. Leur vision est ancrée dans une expérience traumatique qui est celle de l'exil. D'emblée, ils se vivent comme des pièces rapportées dans un monde qui projette sur eux des idées globalisantes. Ainsi, les angoisses des auteurs sont si perceptibles que créer un personnage devient pour eux une façon d'objectiver leur propre identité.

\section{Le refus du despotisme de la création}

La construction du personnage représente pour les dramaturges africains une double difficulté. Créer un personnage est vécu comme un acte despotique qui reproduit la violence avec laquelle l'histoire s'est acharnée à définir l'Afrique. De plus, ce théâtre décrit des sujets sans identités que l'écriture doit présenter comme inachevés.

La distribution des personnages elle-même est problématique. Chez Koulsy Lamko, dans Tout bas... si bas, chaque personnage est accompagné d'un commentaire qui correspond à une note d'intention plus ou moins délayée. Mais au lieu d'indiquer précisément le caractère des personnages, l'auteur sème le doute :

Le reporter : Que vient-il faire dans cette aventure? Pour que l'histoire fonctionne,

il fallait un élément perturbateur de l'équilibre initial. ${ }^{18}$

La présence du reporter est tout à fait fonctionnelle et ne s'explique que par les besoins de la progression narrative. Ce renoncement à construire des personnages aux individualités définies est repris par Koffi Kwahulé qui ne présente pas ses personnages et les laisse émerger d'un flou énigmatique. Dans Big shoot, la didascalie liminaire indique simplement « deux hommes ». Dans Jaz une courte didascalie présente le seul personnage: «Une femme. Le crâne rasé peut-être. Nue peut-être ${ }^{19}$ ». L'emploi récurrent du "peut-être » affiche une hésitation, comme si l'auteur se refusait à détenir une définition de son personnage.

Qui est le personnage qui narre l'histoire de Jaz? Du début à la fin, on hésite. Son amie? Un voisin? On apprend à la fin que c'est elle-même qui se raconte et qui pourtant affirme à plusieurs reprises, comme un refrain, convulsivement : " Non non non, je ne suis pas ici pour parler de moi, mais de Jaz ${ }^{20} »$. Le personnage est menacé par le dédoublement, elle affirme à la fin: "Jaz ce n'est plus moi. Jaz. Oui Jaz. On m'a toujours appelée ainsi ». Le personnage est extérieur à lui-même et se regarde dans un recul dépersonnalisant. Personnage en creux où s'inscrit la violence du monde, le sujet contient l'autre. Le personnage n'a pas les clés de lui-même. Kwahulé confiait à Nabil El Azan qui lui demandait simplement : «qui est Jaz, comment est-elle?»: « Elle est ce 
que toi, metteur en scène, voudras qu'elle soit. Elle est blanche, noire, ou jaune... elle a vingt, trente ou quarante ans ${ }^{21}$ ». L'écriture de Kwahulé, comme l'écrit Nabil El Azan est celle de "l'incomplétude ». Sans doute est-ce aussi la propriété du théâtre, et l'on peut voir ici l'une des raison du choix de cette forme pour des auteurs de l'identité. L'écriture ne fixe rien. La suprématie d'un auteur tout puissant est refusée. Le théâtre devient donc « ce lieu unique où les sans-identité ont droit de cité22 ".

De même, la démission de l'auteur qui passe le pouvoir au metteur en scène permet de rejetter la responsabilité du sens de l'oeuvre. Un nouveau sujet intervient dans la construction de l'identité, et sa vision est capitale. Les mises en scènes peuvent être aussi variées qu'il y a de lectures du texte. L'auteur refuse de finir son texte, et par làmême il refuse de définir le sujet qu'il invente cependant.

\title{
Agregats de soi
}

\author{
«Ce qui peut nous identifier ce n'est pas la façon \\ dont nous fabriquerions une autre parole \\ affirmative, une autre définition de nous-mêmes, \\ mais le refus de la définition ${ }^{23}$. " \\ Kossi Efoui
} leur arrivée en Europe comme un exil. Cet exil est décrit dans Village fou ou les Déconnards, de Kwahulé, Monsieur vit seul dans une petite mansarde, les fantôme de son village natal le hantent et viennent peupler sa solitude de souvenirs; tel Krapp dans La dernière Bande de Beckett, il glisse des cassettes dans des magnétophones et plonge dans un univers passé. De même, ces dramaturges africains sont nourris de cultures africaines dans un monde européen. Leur situation d'exil est à la fois un éloignement et une richesse : leur identité est aussi d'être doubles, tendus entre deux pôles. Kossi Efoui nomme cette identité de la dualité «identité de carrefour », écrit Sylvie Chalaye, autrement dit une identité ouverte au monde. Dans un colloque consacré à la notion d' "entre-deux» dans les dramaturgies africaines à Rennes, Mercédès Fouda, écrivain camerounaise, évoque un entre deux spatial tendu dans un axe nord-sud, un entre deux culturel et temporel entre la période coloniale et postcoloniale. Ce brassage aboutit à une identitée faite d'agrégats, d'ajouts, et non pas à une identité en un bloc. Marcel Zang en dresse une image avec le personnage d'Imago dans L'Exilé :

Je n'ai pas de chez moi, je n'ai pas de pays, je n'ai pas de terre [...] Je veux bien reconnaître certains liens avec l'Afrique, je ne peux le nier, mais mon identité avec l'Afrique est une identité parmi d'autres, parmi des centaines d'autres qui n'ont rien à voir avec l'Afrique. Et à supposer que j'aie une identité, ce ne peut être que cette somme d'identités diverses. ${ }^{24}$

Cette "somme d'identités diverses" permet de battre en brèche cette notion terrifiante de l'identité. Elle apparaît ici comme la vision de soi-même appartenant à une société qui permet de se définir. L'identité telle que la conçoivent certaines sociétés qui se fantasmes puissantes et unies n'est donc qu'un « intégrisme » reposant sur une prétendue " authenticité culturelle », c'est ainsi du moins que l'analyse Kossi Efoui dans la préface de l'Entre-deux rêves de Pitagaba. Elle est ici battue en brèche par l'idée d'agrégat d'expériences et de coutumes adoptées. C'est ainsi que le sujet de ces pièces est résolument dénué d'identité, qu'il n'est pas un être fini, mais fondamentalement 
mouvant, évolutif. Mouvant, car en perpétuel changement, en pleine métamorphose, tel Makiadi qui traverse l'espace et le temps en changeant d'apparence, le sujet est également perpétuellement pris dans une dynamique spatiale. Renoncer à la recherche d'une identité finie est donc un moyen d'essayer de faire taire ce complexe d'infériorité africain. L'africain attend maintenant sa reconnaissance comme un égal des autres peuples. Le théâtre apparaît donc comme le lieu d'une nouvelle présence africaine. Ces dramaturgies convoquent le public dans un face à face identitaire.

\section{La lutte cathartique}

32 A l'entrée de la Comédie de Saint-Etienne où est créée Catharsis, la dernière pièce de Gustave Akakpo, dans une mise en scène de Jean-Claude Berutti, retentit une annonce :

Mesdames, messieurs, le lieu dans lequel vous allez entrer n'est pas un théâtre mais un cimetière qui vous envahira de sa brume et de sa puanteur, on y entre pieds nus. Ce cimetière est situé dans un pays où l'on se bat, où la guerre entre les tribus voisines fait rage depuis des générations, il faudra prendre part à cette guerre en vous munissant de kalachnikov avant d'entrer. A chaque fois que vous entendrez la rumeur d'un bombardement, brandissez-les en criant.

Le ton est donné, on cherche à faire du théâtre un lieu participatif où le spectateur agit au coeur du spectacle. La brume envahit toute la salle, on descend à travers les marches en tâtonnant, on discerne que personne n'est assis. La masse hésitante du public se rend sur scène, dans une arène où la pente permet aux spectateurs de s'asseoir juste au dessus de la fosse où est installé le décor, pentes garnies de tissus africains, de coussins, même d'une rangée de bancs, sous les rambardes. Deux entrées par un escalier. Scène ovale, sur du sable, coulisses sous les gradins, basses, dans lesquelles on entre en rampant. Deux chaises face à face, couvertes d'une peau de bête. Au-dessus des gradins, des guirlandes de lumières, pointant à travers le brouillard. De forts projecteurs apparaissent de plus en plus puissants à mesure que la fumée s'évapore. Un comédien accueille les spectateurs, "Bienvenue, soyez les bienvenus, installez-vous", et indistinctement, sans qu'on ait pu le prévoir, le spectacle commence comme s'il n'était pas écrit mais improvisé, avec cette même spontanéité qu'ont les griots quand ils passent dans le monde du conte. La structure imaginée par Jean-Claude Berutti met au centre du spectacle l'interaction entre public et comédiens qui habitent le même espace. Le spectateur, à qui on a distribué son accessoire, une kalachnikov, joue comme les comédiens, il a le rôle d'un guerrier de cet endroit d'Afrique. Comédiens et spectateurs assistent à la même réalité. Tous deux connaissent leur texte (cris, coups de feu des spectateurs). Les nombreuses adresses au public cherchent à le sortir de la passivité : le photographe demande, par exemple, à un spectateur de lui tenir son pied. Mais ce processus va plus loin, au moment où Ellè décide de danser le rituel, elle a besoin d'un Gardien de l'Oracle, et celui-ci vient d'être blessé d'une balle. Pour éviter que son fils n'ait à le faire elle propose :

(Désignant le public) Il n'y a qu'à demander à l'un d'entre eux, au lieu de nous regarder, qu'ils servent à quelque chose! (Au public) Un volontaire pour mener le rituel, allons, quelqu'un qui a des couilles! Personne n'a des couilles dans le rituel de la Reine-Mère ?25

Les comédiens passent alors dans le public, profitant de la proximité qu'établit la scène ; le public effarouché tremble de devoir descendre sur scène, jusqu'à ce que le spectacle enchaîne, ayant pris le risque d'une intervention d'un spectateur audacieux. Akakpo 
construit un jeu entre texte et improvisation, au confluent de deux cultures, celle de l'oralité et celle du texte, il laisse au public la possibilité de réagir. Le spectacle est au confluent d'une représentation de théâtre contemporain et d'une représentation traditionnelle africaine telle qu'on en a l'image.

Le titre Catharsis assume le fantasme d'un théâtre communautaire. La manière d'impliquer de la même façon acteur et public dans l'action et de reprendre un dispositif circulaire se veut un moyen de souder les deux entités théâtrales. Mais pour se recréer, la communauté doit d'abord purger la violence qui a opposé les peuples. Le texte décrit la violence et la rancœur d'un auteur africain envers le public occidental. La confrontation existe des deux côtés, du côté du public, qui brandit les kalachnikovs, et des acteurs qui insultent parfois le public, l'accusant de "ne pas avoir de couilles». Violences passées des « étrangers du Nord-là-bas » qui ont imposé leurs lois, « Enfants réduits en esclavage? Corps pillés ? C'est un crime. Un crime contre l'humanité26 Jouer quelques générations après devant ces mêmes «étrangers du Nord » ne peut être libre de présupposés. Ce mouvement d'acceptation de l'africain ne va pas sans une déstabilisation du spectateur, ébranlé par le spectacle d'un pays détruit par la guerre, les génocides, et où règne la prostitution. Cathartique, cette pièce l'est assurément. Elle propose un règlement de comptes, une bagarre entre deux peuples qui oblige l'africain à se relever et l'européen à se défendre (à se justifier ?). Le rituel de la pièce est un rituel de purification des rapports Europe / Afrique Après cette confrontation peut naître l'ouverture à l'autre. La reconnaissance mutuelle, mais aussi la prise de conscience que les opposés sont peut-être plus proches qu'on ne le croit et que la crise identitaire qui occupe les personnages sur scène correspond tout aussi bien à un mal européen. Ainsi Gustave Akakpo rappelle-t-il que :

La pièce n'a pas une parole en sens unique juste à l'endroit des Africains mais [...] elle fait écho à la crise d'identité d'une jeunesse européenne, d'une jeunesse tout court, en panne de véritables modèles dont elle a besoin pour façonner son acte d'existence. ${ }^{27}$

Les sujets qui donnent vie à ce théâtre contribuent donc à détruire la notion d'identité culturelle. Le concept "d'authenticité culturelle », qualifié d' «intégrisme » par Kossi Efoui dans la préface de L'entre-deux rêves de Pitagaba, force l'individu à se définir non plus par son passé, son milieu, son héritage culturel, mais par son action et sa confrontation au monde. Il y a dans cette redéfinition une ouverture de l'Afrique et une condamnation de la posture de repli défendue par les générations précédentes, au nom de leur "africanité ». Le personnage n'étant jamais complètement défini par son auteur, il peut donc recueillir de multiples identités, celles que projettera chaque lecteur, chaque metteur en scène. Il peut être incarné par n'importe quel acteur et s'adresse à des spectateurs d'origines différentes. Le théâtre africain, autrefois parqué dans un mélange de conte et de couleur locale, a su se faire profondément contemporain. Le sujet africain, après s'être exposé au monde, attend sa reconnaissance, il reste incomplet, en attente d'un devenir, allégorie d'un monde en crise d'identité. L'épitaphe de La fable du cloître des cimetières, extraite de L'herbe rouge de Boris Vian, le rappelle :

Il marchait sans penser à rien, sans rien voir. Il éprouvait seulement une impression

d'attente. Quelque chose allait se passer, bientôt. 


\section{BIBLIOGRAPHIE}

Corpus :

AKAKPO, Gustave, Catharsis, Carnières, Lansman, 2006.

EFOUI, Kossi, L'entre-deux rêves de Pitagaba, Paris, Acoria éditions, 2000.

KWAHULE, Koffi, La dame du café d'en face/Jaz, Paris, Éditions théâtrales, 1998.

- Big Shoot/ P'tite souillure, Paris, Éditions théâtrales, 2000.

LAMKO, Koulsy, Tout bas... si bas, Carnières, Lansman, 1995.

MAKHELE, Caya, La fable du cloître des cimetières, Paris, L'Harmattan, 1995.

- Les travaux d'Ariane, Acoria/ Asphalte, Paris, 2006.

ZANG, Marcel, L'Exilé, Arles, Actes Sud Papiers, 2002.

Ouvrages théoriques:

CHALAYE, Sylvie (dir.), Nouvelles dramaturgies d'Afrique noire francophone, Rennes, Presses Universitaires de Rennes, 2004.

CHALAYE, Sylvie, Afrique noire et dramaturgies contemporaines : Le syndrome Frankenstein, Paris, Éditions théâtrales, passages francophones, 2004.

CHALAYE, Sylvie, Dramaturgies africaines d'aujourd'hui en dix parcours, Carnières, Lansman, 2001.

KWAHULE, Koffi, Pour une critique du théâtre ivoirien contemporain, L'Harmattan, 1997.

SCHERER, Jacques, Le théâtre en Afrique noire francophone, Paris, PUF, 1992.

Revues :

Numéros de la revue Africultures:

Monde noir et scènes contemporaines, Paris, L'Harmattan, n50, septembre 2002.

- Acteurs noirs, Paris, L’Harmattan, n²7 avril 2000.

- Théâtres en écritures, Paris, L'Harmattan ํ10sep 1998.

Numéros de la revue Théâtre/public:

- Les ombres de la rampe: les comédiens noirs de la scène française, n¹72, Gennevilliers, 2004.

- Afrique noire: écritures contemporaines, $\mathrm{n}^{\circ}$ 158, Gennevilliers 2000.

Sites Internet :

http://africultures.com/ [consulté le 11/12/07]

Échange de mails entre Jean-Claude Berutti et Gustave Akakpo à propos de Catharsis : https:// www.theatre-contemporain.net/spectacles/Catharsis/ensavoirplus/ [consulté le 11/12/07]

\section{NOTES}

1. Caya Makhélé, La fable du cloître des cimetières, Paris, L'Harmattan, 1995, p. 18.

2. Ibidem. 
3. Ibid., p. 21.

4. Ibid., p. 19.

5. Caya Makhélé, Les Travaux d'Ariane, Paris Acoria/ Asphalte, 2006, p. 9.

6. Caya Makhélé, La fable du cloitre des cimetières, op. cit., p. 23.

7. Par «titre didascalique " j'entends titre donné à une scène explicitant l'action à venir ou rappelant l'étape de l'histoire à laquelle on assiste.

8. Caya Makhélé, La fable du cloître des cimetières, op. cit., p. 34.

9. Ibid., p. 55.

10. Ibid., p. 52.

11. Koffi Kwahulé, Big Shoot, Paris, Éditions théâtrales, 2000, p. 28.

12. Ibid., p. 12.

13. Ibidem.

14. Ibid., p. 21.

15. Ibid., p. 9.

16. Ibid., p. 17.

17. Ibid., p. 45.

18. Koulsy Lamko, Tout bas... si bas, Carnières, Éditions Lansman, 1995, p. 6.

19. Koffi Kwahulé, Jaz, Éditions théâtrales, Paris, 1998, p. 57.

20. Ibid., p. 24.

21. Nabil El Azan, préface à La Dame du café d'en face et Jaz, Koffi Kwahulé, Paris, Éditions théâtrales, 1998, p. 6.

22. Ibidem.

23. Kossi Efoui, entretien avec Sylvie Chalaye, Afrique noire: écritures contemporaines, Afrique noire: écritures contemporaines, Gennevilliers, Théâtre/Public n¹58, mars-avril 2001, p. 81.

24. Marcel Zang, L'Exilé, Arles, Actes Sud-Papiers, 2002, p. 30.

25. Gustave Akakpo, Catharsis, Carnières, Lansman, 2006, p. 21.

26. Ibid., p. 24.

27. Gustave Akakpo, Dialogue par mails entre l'auteur et le metteur en scène de Catharsis, entre Lomé et Saint-Étienne: https://www.theatre-contemporain.net/spectacles/Catharsis/ ensavoirplus/ [consulté le 11/12/07].

\section{INDEX}

Mots-clés : théâtre contemporain africain, Akakpo (Gustave), Kwahulé (Koffi), Lamko (Koulsy), Makhélé (Kaya) 